\title{
SINGULARLY PERTURBED TELEGRAPH EQUATIONS WITH APPLICATIONS IN THE RANDOM WALK THEORY
}

\author{
JACEK BANASIAK and JANUSZ R. MIKA ${ }^{1}$ \\ University of Natal, Dept. of Mathematics and Applied Mathematics \\ Private Bag X10, Dalbridge 4014, Durban \\ South Africa
}

(Received March, 1996; Revised May, 1997)

In the paper we analyze singularly perturbed telegraph systems applying the newly developed compressed asymptotic method and show that the diffusion equation is an asymptotic limit of singularly perturbed telegraph system of equations. The results are applied to the random walk theory for which the relationship between correlated and uncorrelated random walks is explained in asymptotic terms.

Key words: Random Walk, Telegraph Equations, Singular Perturbation, Asymptotic Analysis, Diffusion Approximation.

AMS subject classifications: $35 \mathrm{~B} 25,60 \mathrm{~J} 15,35 \mathrm{~L} 50$.

\section{Introduction}

An asymptotic equivalence between uncorrelated and correlated random walks (or between the diffusion and telegraph equations) has been studied by many authors (see e.g. [14], a survey in [16] or recent paper [7]). The results can be divided into two streams: in the first one the probabilistic aspect of the problem is of primary interest and the results for the differential equations are obtained as a by-product and in the second one the situation is reversed. Our paper belongs to the second stream. We combine recent advances in the asymptotic analysis of kinetic equations with an observation that the system of telegraph equation is in fact a simplified Boltzmann equation (which was probably pointed out first by Kac [8]) to provide a unified treatment of various singularly perturbed telegraph systems showing their asymptotic equivalence with a diffusion equation.

In most cases known to us the asymptotic analysis is carried out for the second order telegraph equation. However, it is known that in the continuous limit of the correlated random walk one obtains a system of equations which can be reduced to the second order equation only for a restricted class of coefficients. Thus, the confinement to the second order telegraph equation seems to limit considerably the generali-

\footnotetext{
${ }^{1}$ Current address: Institute of Atomic Energy, Świerk, 05-400 Otwock, Poland and Institute of Mathematics, Technical University of Łódź, Poland.
} 
ty of the results so that in this paper we consider the systems of telegraph equations with variable coefficients.

A well-known result is that the correlated random walk tends to an uncorrelated random walk if the strength of correlations goes to zero but at the same time the speed of particles tends to infinity in such a way that

$$
\text { strength of correlations } \times(\text { speed of particles })^{2}=O(1),
$$

(see e.g. [7] and the references therein). We recover this result and, introducing the initial layer, extend it to hold uniformly in time for any bounded time interval $[0, T]$. Moreover, we shall show that the diffusion approximation is also valid under the sole assumption that the strength of correlations tends to zero allowing the speed of particles to remain finite. In this case, the error of approximation turns out to be much smaller than in the previous case.

In the analysis the crucial role is played by the recently developed compressed method (which is a modification of the Chapman-Enskog method, well-known in kinetic theory). The detailed description of the compressed method can be found in [11]. The reader is also advised to consult the relevant references cited in the book (particularly, $[1,3,4,9,10,12])$.

\section{Basic Notions from Random Walk Theory}

In this section, we introduce relevant definitions and formulas from random walk theory. Since, however, our primary aim is the singular perturbation analysis, we confine ourselves to a simple, one dimensional free-space case, as presented e.g. in [16].

Let us assume that each particle moves on the real line, with common probability $p(x)$ at position $x$ to the right and to the left by the distance $\delta$ every time interval $\theta$. We define $v(x, t)$ as the probability of finding a particle at the position $x$ and time $t$ if it starts at $x=0$ and $t=0$. If we denote $\sigma(x)=2 p(x)$, then the probability that the particle rests at each step is $1-\sigma(x)$. The function $v$ satisfies the forward Kolmogorov equation

$$
v(x, t+\theta)=(1-\sigma(x)) v(t, x)+p(x-\delta) v(x-\delta, t)+p(x+\delta) v(x+\delta, t) .
$$

By expanding the functions in (2.1) into Taylor series and passing to the limits as $\delta$, $\theta \rightarrow 0$ in such a way that

$$
D(x):=\lim _{\substack{\delta \rightarrow \infty \\ \theta \rightarrow \infty}} \frac{\sigma(x) \delta^{2}}{\theta}
$$

remains finite, we arrive at the diffusion (Fokker-Planck) equation satisfied by the probability distribution function $v$ :

$$
\partial_{t} v=\frac{1}{2} \partial_{x}^{2}(D v)
$$

Equation (3.2) is to be supplemented by the initial condition

$$
v(x, 0)=\stackrel{\circ}{v} .
$$


The requirement that the diffusion constant $D$ is finite implies that the particle speed

$$
\gamma:=\lim _{\substack{\delta \rightarrow \infty \\ \theta \rightarrow \infty}} \frac{\delta}{\theta}
$$

is infinite. This result clearly indicates that the diffusion equation does not supply an adequate description of random walk. The reason is that the Kolmogorow equation (2.1) refers to an uncorrelated random walk so that remedy this drawback we have to take into account the correlations. There are various ways to do this like, for instance, utilizing the Langevin equation. We will choose the approach of Taylor [15] and Goldstein [6] and introduce two probability distribution functions, separately for particles moving to the right $\psi$, and to the left $\chi$. In a similar way we take $p$ as the probability that a particle persists in moving in the same direction and $q$ as the probability that it changes the direction. The probability that the particle rests at each step is given by $1-\sigma(x)$, where

$$
\sigma(x)=p(x)+q(x)
$$

We assume that $\sigma$ is a Lipschitz continuous function and

$$
0<\sigma_{0} \leq \sigma(x) \leq \sigma_{1}<\infty
$$

for all $x \in \mathbb{R}$.

Under these assumptions, we have the following system of Kolmogorow equations for the functions $\psi$ and $\chi$

$$
\begin{aligned}
\psi(x, t+\theta)=(1- & p(x)-q(x)) \psi(x, t)+p(x-\delta) \psi(x-\delta, t) \\
& +q(x-\delta) \chi(x-\delta, t) \\
\chi(x, t+\theta)=(1- & p(x)-q(x)) \chi(x, t)+p(x+\delta) \chi(x+\delta, t) \\
& +q(x+\delta) \chi(x+\delta, t) .
\end{aligned}
$$

In the presence of correlations we expect that $p$ is approaching $\sigma$ and $q$ is approaching 0 when $\theta \rightarrow 0$ and relation (2.6) holds. Therefore, it is reasonable to take $p$ and $q$ in the following form:

$$
p(x)=\sigma(x)-\lambda(x) \theta+O\left(\theta^{2}\right), q(x)=\lambda(x) \theta+O\left(\theta^{2}\right)
$$

where $\lambda$ is the rate of reversal of direction and is related to the strength of correlations in the system. We assume that $\lambda$ is a Lipschitz continuous function satisfying

$$
0<\lambda_{0} \leq \lambda(x) \geq \lambda_{1}<\infty
$$

for all $x \in \mathbb{R}$.

Expanding the functions $\psi$ and $\chi$ in Taylor series, using Equation (2.10) and letting $\delta, \theta \rightarrow 0$, in such a way that $\gamma$ defined in Equation (2.5) remains finite, we obtain the system of equations 


$$
\begin{aligned}
& \partial_{t} \psi=-\gamma \partial_{x}(\sigma \psi)-\lambda \psi+\lambda \chi \\
& \partial_{t} \chi=-\gamma \partial_{x}(\sigma \chi)+\lambda \psi-\lambda \chi
\end{aligned}
$$

It is convenient to introduce two new functions

$$
v(t, x)=\psi(t, x)+\chi(t, x)
$$

and

$$
w(t, x)=\psi(t, x)=\chi(t, x)
$$

where $v$ is the density and $w$ is the net (to the right) current of the particles. Now adding and subtracting the equations in (2.12) we obtain the telegraph system:

$$
\begin{gathered}
\partial_{t} v+\gamma \partial_{x}(\sigma w)=0 \\
\partial_{t} w+\gamma \partial_{x}(\sigma v)+2 \lambda w=0
\end{gathered}
$$

System (2.15) is supplemented with the initial conditions

$$
v(x, p)=\stackrel{\circ}{v}, w(x, 0)=\stackrel{\circ}{w} .
$$

Due to the presence of variable coefficients in system (2.15) it is convenient to consider it as an evolution equation

where

$$
\partial_{t}\left[\begin{array}{c}
v \\
w
\end{array}\right]=\gamma \varphi\left[\begin{array}{c}
v \\
w
\end{array}\right]+2 \lambda e\left[\begin{array}{l}
v \\
w
\end{array}\right]
$$

$$
\varphi=\left[\begin{array}{cc}
0 & \partial_{x} \sigma \\
\partial_{x} \sigma & 0
\end{array}\right] \text { and } \mathcal{C}=\left[\begin{array}{cc}
0 & 0 \\
0 & -1
\end{array}\right]
$$

in the Hilbert space $\mathfrak{H}_{\sigma}=L_{2}(\mathbb{R}) \times L_{2}(\mathbb{R})$ with the following norm:

$$
\|(v, w)\|_{\sigma}^{2}=\int_{\mathbb{R}}|v(x)|^{2} \sigma(x) d x+\int_{\mathbb{R}}|w(x)|^{2} \sigma(x) d x .
$$

This norm is equivalent to the natural one by virtue of Equation (2.7).

In a similar manner we define norms in the Sobolev spaces used throughout the paper; these spaces will be denoted by $W_{2, \sigma}^{k}$.

It then follows that the operator $\varphi^{2}$ defined on the domain $D(\varphi)=W_{2, \sigma}^{1}(\mathbb{R}) \times$ $W_{2, \sigma}^{1}(\mathbb{R})$ is maximal dissipative and hence it generates a semigroup of contractions denoted by $\left(G_{\varphi}(t)\right)_{t \geq 0}$. Since the operator $2 \lambda C$ is bounded, the operator $\mathcal{\sigma}:=\varphi+2 \lambda e$ also generates a semigroup of contractions, say, $\left(G_{\mathcal{G}}(t)\right)_{t} \geq 0$. Hence the Cauchy problem related to Equation (2.17) is solvable. 


\section{Setting the Problem}

If the correlations are weak, that is, the coefficient $\lambda$ is large, it is natural to expect that the probability density $v$ of the correlated random walk should be close to the probability density obtained by solving diffusion equation (2.3) of the uncorrelated random walk.

A well-known result in this field is that if $\lambda$ is independent of $x$ and $\lambda, \gamma \rightarrow \infty$ in such a way that $\gamma^{2} / \lambda$ remains finite and non-zero, then system (2.15) reduces to the diffusion-type equation:

$$
\partial_{t} v=\frac{1}{2} \partial_{x}\left(\sqrt{D} \partial_{x}(\sqrt{D} v)\right)
$$

for $v$, where

$$
\sqrt{D}:=\lim _{\substack{\lambda \rightarrow \infty \\ \gamma \rightarrow \infty}} \frac{\gamma}{\sqrt{\lambda} \sigma}
$$

We note that Equation (3.1) coincides with Equation (2.3) only when $D$ is constant. The proof of convergence can be found in e.g. [5, 7].

In this paper we show that the solution to the diffusion equation approximates that of the correlated random walk and provide estimates of th incurred error. Moreover, we shall show that the diffusion approximation is also valid when we let $\lambda \rightarrow \infty$ and assuming that $\gamma$ is finite. This seems to be by far more acceptable from the physical point of view, since there are no reasons for the signal to travel at infinite speed. All these will be done in a general setting of the singular perturbation theory developed originally for kinetic equations in [11]. In the spirit, of the theory we normalize the relevant coefficients in system $(2.15)$ by introducing a small positive parameter $\epsilon$, independent of $x$.

To cover both types of behavior of $\gamma$ we consider two separate cases.

First case: With

$$
\gamma=\frac{\widehat{\gamma}}{\epsilon}, \quad \lambda=\frac{\widehat{\lambda}}{\epsilon^{2}}
$$

Equation (2.17) takes the form:

$$
\partial_{t}\left[\begin{array}{c}
v \\
w
\end{array}\right]=\frac{\widehat{\gamma}}{\epsilon} \varphi\left[\begin{array}{c}
v \\
w
\end{array}\right]+\frac{2 \widehat{\lambda}}{\epsilon} \mathrm{e}\left[\begin{array}{c}
v \\
w
\end{array}\right] .
$$

Second case: For

$$
\lambda=\frac{\widehat{\lambda}}{\epsilon}
$$

Equation (2.17) is written as

$$
\partial_{t}\left[\begin{array}{c}
v \\
w
\end{array}\right]=\gamma \varphi\left[\begin{array}{c}
v \\
w
\end{array}\right]+\frac{2 \hat{\lambda}}{\epsilon}\left[\begin{array}{c}
v \\
w
\end{array}\right] .
$$

We note that mathematically speaking, forms (3.3) and (3.5) are equivalent as rescaling time $t \rightarrow \epsilon t$, discussed in detail in Section 5, transforms (3.3) into (3.5) and, therefore, $t \rightarrow(v(t, \cdot), w(t, \cdot))$ is a trajectory of the former if and only if $t \rightarrow$ $(v(\epsilon t, \cdot), w(\epsilon t, \cdot))$ is the trajectory of the latter. However, as we shall see it later, the 
asymptotic behavior of the trajectories as $\epsilon \rightarrow 0$ are quite different and full comparison is not possible unless some sophisticated asymptotic procedure is used. In this paper we present such a procedure, called the compressed asymptotic procedure, which is a modified version of the well-known Chapman-Enskog procedure in kinetic theory. An exhaustive discussion of various scalings of equations can be found in $[2,11]$.

Basic features of the compressed asymptotic method are:

1. Functions $v$ and $w$ are sought in the form

$$
\begin{aligned}
v(t) & =\bar{v}(t)+\widetilde{v}(\tau), \\
w(t) & =\bar{w}(t)+\widetilde{w}(\tau),
\end{aligned}
$$

where $\tau=t / \epsilon^{2}$ in the first case, and $\tau=t / \epsilon$ in the second case, is the "stretched" time variable necessary to cater for the initial layer phenomena; $\bar{v}, \bar{w}$ and $\widetilde{v}, \widetilde{w}$ are sought independently.

2. Function $\bar{w}$ depends on $t$ only through its dependence on $\bar{v}$, that is, for some function $W$,

$$
\bar{w}(t)=W(\bar{v}(t))
$$

3. Functions $\bar{w}, \widetilde{v}, \widetilde{w}$ are expanded into asymptotic series in $\epsilon$ :

$$
\begin{aligned}
\bar{w}(t) & =\bar{w}_{0}(t)+\epsilon \bar{w}_{1}(t)+\epsilon^{2} \bar{w}_{2}(t)+O\left(\epsilon^{3}\right) \\
& =W_{0}(\bar{v}(t))+\epsilon W_{1}(\bar{v}(t))+\epsilon^{2} W_{2}(\bar{v}(t))+O\left(\epsilon^{3}\right), \\
\widetilde{v}(\tau) & =\widetilde{v}_{0}(\tau)+\epsilon \widetilde{v}_{1}(\tau)+\epsilon^{2} \widetilde{v}_{2}(\tau)+O\left(\epsilon^{3}\right), \\
\widetilde{w}(\tau) & =\widetilde{w}_{0}(\tau)+\epsilon \widetilde{w}_{1}(\tau)+\epsilon^{2} \widetilde{w}_{2}(\tau)+O\left(\epsilon^{3}\right),
\end{aligned}
$$

whereas $\bar{v}$ remains unexpanded and treated as an $O(1)$ function at all levels of approximation.

Remark 3.1: Assumption (3.6) is known in the theory of autonomous differential equation, where very often taking the unknown function as a new independent variable simplifies the considerations. The assumption that $\bar{v}$ is not expanded is then merely a statement of the fact that one cannot expand an independent variable. In our case, however, (3.6) will be satisfied in the asymptotic sense only.

The reason for truncating the expansion at $\epsilon^{2}$ terms is, as will be clear from the formal asymptotic expansion of the next section, that we fail to obtain the diffusion equation for higher order approximations.

\section{Singularly Perturbed Systems: First Case}

In this section we are concerned with the singularly perturbed initial value problem (3.3), which can be written in an expanded form as follows:

$$
\partial_{t} v+\frac{\widehat{\gamma}}{\epsilon} \partial_{x}(\sigma w)=0
$$




$$
\partial_{t} w+\frac{\widehat{\gamma}}{\epsilon} \partial_{x}(\sigma v)+\frac{2 \widehat{\lambda}}{\epsilon} w=0
$$

with the initial values given by

$$
v(0, x)=\stackrel{\circ}{v}, \quad w(0, x)=\stackrel{\circ}{w} .
$$

Inserting (3.7) into (4.1) we obtain the following equations:

$$
\partial_{t} \bar{v}=-\frac{1}{\epsilon} \widehat{\gamma} \partial_{x}\left(\sigma \bar{w}_{0}\right)-\widehat{\gamma} \partial_{x}\left(\sigma \bar{w}_{1}\right)-\epsilon \widehat{\gamma} \partial_{x}\left(\sigma \bar{w}_{2}\right)-\ldots
$$

Denoting $W_{j}^{\prime}=\frac{d W_{j}}{d \bar{v}}$ for $j=0,1, \ldots$, we have

$$
\begin{aligned}
& W_{0}^{\prime} \partial_{t} \bar{v}+\epsilon W_{1}^{\prime} \partial_{t} \bar{v}+\epsilon^{2} W_{1}^{\prime} \partial_{t} \bar{v}+\ldots+\frac{1}{\epsilon} \widehat{\gamma} \partial_{x}(\sigma \bar{v}) \\
& +2 \hat{\lambda}\left(\frac{1}{\epsilon^{2}} W_{0}(\bar{v})+\frac{1}{\epsilon} W_{1}(\bar{v})+W_{s}(\bar{v})\right)+\ldots=0 .
\end{aligned}
$$

Inserting expansion (4.3) into (4.4) and comparing the coefficients at the same powers $\epsilon$ we get:

$$
\begin{aligned}
& O\left(\epsilon^{-2}\right): w_{0}=0 \\
& O\left(\epsilon^{-1}\right): w_{1}=-\frac{\widehat{\gamma}}{2 \widehat{\lambda}} \partial_{x}(\sigma \bar{v}) \\
& O\left(\epsilon^{0}\right): w_{2}=0 .
\end{aligned}
$$

Going back to (4.3) we obtain the following diffusion equation:

$$
\partial_{t} \bar{v}=\frac{\gamma^{2}}{2} \partial_{x}\left(\frac{\sigma}{\lambda} \partial_{x}(\sigma \bar{v})\right)
$$

which coincides with Equation (3.1) if $\lambda$ is independent of $x$. Here we used the fact that $\widehat{\gamma}^{2} / \widehat{\lambda}=\gamma^{2} / \lambda$.

To obtain the initial layer terms and the appropriate initial conditions for Equation (4.8) we insert Equations (3.8) and (3.9) into Equation (4.1) to obtain

$$
\partial_{\tau} \widetilde{v}_{0}+\epsilon \partial_{\tau} \widetilde{v}_{1}+\epsilon^{2} \partial_{\tau} \widetilde{v}_{2} \ldots+\epsilon \widehat{\gamma}\left(\partial_{x}\left(\sigma \widetilde{w}_{0}\right)+\epsilon \partial_{x}\left(\sigma \widetilde{w}_{1}\right)+\epsilon^{2} \partial_{x}\left(\sigma \widetilde{w}_{2}\right)+\ldots\right)=0
$$

and

$$
\begin{gathered}
\partial_{\tau} \widetilde{w}_{0}+\epsilon \partial_{\tau} \widetilde{w}_{1}+\epsilon^{2} \partial_{\tau} \widetilde{w}_{2} \ldots+\epsilon \widehat{\gamma}\left(\partial_{x}\left(\sigma \widetilde{v}_{0}\right)+\epsilon \partial_{x}\left(\sigma \widetilde{v}_{1}\right)+\epsilon^{2} \partial_{x}\left(\sigma \widetilde{v}_{2}\right)+\ldots\right) \\
=2 \widehat{\lambda}\left(\widetilde{w}_{0}+\epsilon \widetilde{w}_{1}+\epsilon^{2} \widetilde{w}_{2} \ldots\right)
\end{gathered}
$$

Comparing coefficients at the same powers of $\epsilon$ we obtain the following set of equations:

$$
\begin{aligned}
& O\left(\epsilon^{0}\right): \partial_{\tau} \widetilde{v}_{0}=0 \\
& \partial_{\tau} \widetilde{w}_{0}=-2 \hat{\lambda} \widetilde{w}_{0},
\end{aligned}
$$




$$
\begin{aligned}
O\left(\epsilon^{1}\right): \partial_{\tau} \widetilde{v}_{1} & =-\widehat{\gamma} \partial_{x}\left(\sigma \widetilde{w}_{0}\right) \\
\partial_{\tau} \widetilde{w}_{1} & =-2 \widehat{\lambda} \widetilde{w}_{1} \\
O\left(\epsilon^{2}\right): \partial_{\tau} \widetilde{v}_{2} & =-\widehat{\gamma} \partial_{x}\left(\sigma \widetilde{w}_{1}\right) \\
\partial_{\tau} \widetilde{w}_{2} & =-2 \widehat{\lambda} \widetilde{w}_{2}-\widehat{\gamma} \partial_{x}\left(\sigma \widetilde{v}_{1}\right) .
\end{aligned}
$$

Recall that the solutions of Equations (4.11) represent the initial layer part and must vanish exponentially at infinity. With this, we obtain that

$$
\begin{aligned}
\widetilde{v}_{0} & \equiv 0 \\
\widetilde{w}_{0}(\tau) & =\widetilde{w}_{0}(0) e^{-2 \hat{\lambda} \tau} \\
\tilde{v}_{1}(\tau) & =\widehat{\gamma} \partial_{x}\left(\frac{\sigma}{2 \widehat{\lambda}} e^{-2 \hat{\lambda} \tau} \widetilde{w}_{0}(0)\right) \\
\widetilde{w}_{1}(\tau) & =\widetilde{w}_{1}(0) e^{-2 \hat{\lambda} \tau} \\
\widetilde{v}_{2}(\tau) & =\widehat{\gamma} \partial_{x}\left(\frac{\sigma}{2 \widehat{\lambda}} e^{-2 \hat{\lambda} \tau} \widetilde{w}_{1}(0)\right)
\end{aligned}
$$

We omit writing the explicit formula for $\widetilde{w}_{2}$ as it is not needed in the sequel. To determine the initial values for Equations (4.8) and (4.11), we balance the initial values

$$
\stackrel{\circ}{v}=\bar{v}(0)+\epsilon \widetilde{v}_{1}(0)+\epsilon^{2} \widetilde{v}_{2}(0)
$$

and

$$
\stackrel{\circ}{w}=\widetilde{w}_{0}(0)+\epsilon\left(\bar{w}_{1}(0)+\widetilde{w}_{1}(0)\right)+\epsilon^{2} \widetilde{w}_{2}(0)
$$

where we have to remember that Equation (4.13) defines the expansion of $\bar{v}(0)$. Taking that into account, we obtain from Equations (4.14) and (4.12) that

$$
\begin{aligned}
& \tilde{w}_{0}(0)=\stackrel{\circ}{w} \\
& \tilde{v}_{1}(0)=\widehat{\gamma} \partial_{x}\left(\frac{\sigma}{2 \widehat{\lambda}} \stackrel{\circ}{w}\right) \\
& \widetilde{w}_{1}(0)=-\bar{w}_{1}(0)=\frac{\widehat{\gamma}}{2 \widehat{\lambda}} \partial_{x}(\sigma \bar{v}(0)) \\
& \tilde{v}_{2}(0)=\frac{\widehat{\gamma}^{2}}{4} \partial_{x}\left(\frac{\sigma}{\widehat{\lambda}^{2}} \partial_{x}(\sigma \bar{v}(0))\right) \\
& \tilde{w}_{2}(0)=0
\end{aligned}
$$


and for $\bar{v}(0)$,

$$
\bar{v}(0)=\stackrel{\circ}{v}-\epsilon \widehat{\gamma} \partial_{x}\left(\frac{\sigma}{2 \widehat{\lambda}} \stackrel{\circ}{w}\right)-\epsilon \frac{\widehat{\gamma}^{2}}{4} \partial_{x}\left(\frac{\sigma}{\widehat{\lambda}^{2}} \partial_{x}(\sigma \bar{v}(0))\right)
$$

The last equation is a second order differential equation and it will be replaced by an approximate expression for the initial value for $\bar{v}(0)$ :

$$
\bar{v}^{(2)}(0)=\stackrel{\circ}{v}-\epsilon \widehat{\gamma} \partial_{x}\left(\frac{\sigma}{2 \widehat{\lambda}} \stackrel{\circ}{w}\right)-\epsilon^{2} \frac{\gamma^{2}}{4} \partial_{x}\left(\frac{\sigma}{\widehat{\lambda}^{2}} \partial_{x}(\sigma \stackrel{o}{v})\right)
$$

The solution of the initial value problem (4.8), (4.21) will be denoted accordingly by $\bar{v}^{(2)} ; \bar{v}(0)$ in Equations $(4.17),(4.18) \bar{v}(0)$ is replaced by $\bar{v}^{(2)}(0)$.

The error of the approximation is defined by:

$$
\begin{gathered}
y\left(t=v(t)-\bar{v}^{(2)}(t)-\epsilon \widetilde{v}_{1}\left(t / \epsilon^{2}\right)-\epsilon^{2} \widetilde{v}_{2}\left(t / \epsilon^{2}\right)\right. \\
z(t)=w(t)-\widetilde{w}_{0}\left(t / \epsilon^{2}\right)-\epsilon\left(\widetilde{w}_{1}\left(t / \epsilon^{2}\right)+\bar{w}\right)-\epsilon^{2} \widetilde{w}_{2}\left(t / \epsilon^{2}\right) .
\end{gathered}
$$

To find the estimates of the error we insert (formally) $v$ and $w$, as obtained from Equations (4.22) and (4.23), into Equations (4.1). After algebraic manipulations utilizing Equations (4.5)-(4.7) and (4.11) we obtain:

$$
\begin{gathered}
\partial_{t} y+\frac{\widehat{\gamma}}{\epsilon} \partial_{x}(\sigma z)=-\epsilon \partial_{x}\left(\sigma \widetilde{w}_{2}\right) \\
\partial_{t} z+\frac{\widehat{\gamma}}{\epsilon} \partial_{x}(\sigma y)+\frac{2 \widehat{\lambda}}{\epsilon} z=-\epsilon \widehat{\gamma} \partial_{x}\left(\sigma \widetilde{v}_{2}\right)-\epsilon \partial_{t} \bar{w}_{1} .
\end{gathered}
$$

The initial data for this system can be calculated as

$$
\begin{gathered}
y(0)=\epsilon^{3} \frac{\gamma^{2}}{4}\left\{\partial_{x}\left[\frac{\sigma}{\widehat{\lambda}^{2}} \partial_{x}\left(\sigma \widehat{\gamma} \partial_{x}\left(\frac{\sigma \stackrel{o}{w}}{2 \widehat{\lambda}}\right)+\sigma \epsilon \partial_{x}\left(\frac{\sigma}{\widehat{\lambda}^{2}} \partial_{x}(\sigma \stackrel{\circ}{v})\right)\right)\right]\right\} \\
z(0)=0
\end{gathered}
$$

We will need the following result:

Proposition 4.1: The operator $\mathscr{D}$ defined by

$$
\Im u=\frac{\gamma^{2}}{2} \partial_{x}\left(\frac{\sigma}{\lambda} \partial_{x}(\sigma u)\right)
$$

with the domain

$$
D(\mathscr{D})=W_{2}^{2}(\mathbb{R})
$$

generates a uniformly bounded analytic semigroup, say $\left(G_{\text {ๆ }}(t)\right)_{t \geq 0}$, in $L_{2}(\mathbb{R})$.

Proof: As in Section 2 we introduce the weighted space $H_{\sigma}=L_{2}(\mathbb{R}, \sigma d x)$. Clearly, $H_{\sigma}=L_{2}(\mathbb{R})$, both set-theoretically and topologically. Norms on the respective Sobolov spaces are redefined accordingly. Let us consider the bilinear form

$$
a_{\alpha}(\phi, \psi)=\int_{\mathbb{R}} \frac{\sigma}{\lambda} \partial_{x}(\sigma \phi) \partial_{x}(\sigma \bar{\psi}) d x+\alpha(\phi, \psi)_{H_{\sigma}},
$$


where $\alpha$ is a complex constant. Clearly, this form is continuous on $W_{2}^{1}(\mathbb{R})$ and, since the mapping $\phi \rightarrow \sigma \phi$ is an isomorphism on $W_{2}^{1}(\mathbb{R})$, we have for $\operatorname{Re} \alpha>0$ that

$$
\begin{gathered}
\operatorname{Re} a_{\alpha}(\phi, \phi) \geq C^{\prime}\left\|\partial_{x}(\sigma, \phi)\right\|_{H_{\sigma}}^{2}+\operatorname{Re} \alpha\|\phi\|_{H_{\sigma}}^{2} \\
\geq C^{\prime \prime}\left\|\partial_{x} \phi\right\|_{H_{\sigma}}^{2}+\operatorname{Re} \alpha\|\phi\|_{H_{\sigma}}^{2} \geq C\|\phi\|_{W_{2}^{1}(\mathbb{R})}^{2},
\end{gathered}
$$

for some constants $C^{\prime}, C^{\prime \prime}, C$. Hence, $a_{\alpha}$ is coercive on $H_{\sigma}$ and, therefore, the operator $\mathscr{~}$, associated with the bilinear form $a_{0}$ and defined on the domain

$$
D(\mathscr{D})=\left\{u \in W_{2}^{1}(\mathbb{R}), \mathscr{D} u \in L_{2}(\mathbb{R})\right\}
$$

is the generator of an analytic semigroup (see e.g. [5]). Letting $\alpha=0$ in Equation (4.29) we see that $\mathscr{D}$ is dissipative in $H_{\sigma}$, hence, $\left(G_{\mathscr{\sigma}}(t)\right)_{t \geq 0}$ is a uniformly bounded semigroup in $L_{2}(\mathbb{R})$ (and, clearly, a semigroup of contractions in $H_{\sigma}$ ).

By classical results on regularity of variational solutions of elliptic problems we obtain that $D(\mathscr{T})$ is given by Equation (4.28).

For asymptotic estimates we shall need explicit expressions for domains of some powers of $\mathscr{D}$. For the free space we have an easy identification

$$
D\left(\Im^{\alpha}\right)=W_{2}^{2 \alpha}(\mathbb{R})
$$

where $\alpha \in \mathbb{R}$.

We can now formulate and prove the main theorem.

Theorem 4.1: Let

(i) $\quad \sigma$ and $\hat{\lambda}$ satisfy

$$
\begin{aligned}
& 0<\sigma_{0} \leq \sigma(x) \leq \sigma_{1}<\infty \\
& 0<\widehat{\lambda}_{0} \leq \widehat{\lambda}(x) \leq \widehat{\lambda}_{1}<\infty
\end{aligned}
$$

(ii) $\quad \stackrel{\circ}{\stackrel{\circ}{v} \in W_{2}^{4}(\mathbb{R})}$,

(iii) $\stackrel{\circ}{w} \in W_{2}^{3}(\mathbb{R})$.

Then, for each $T, 0 \leq T \leq \infty$, there is a constant $C$ such that the error of approximation $\{y, z\}$ defined in Equations (4.22) and (4.23) satisfies

$$
\|\{y, z\}\|_{L_{2}(\mathbb{R}) \times L_{2}(\mathbb{R})} \leq C \epsilon^{2}
$$

uniformly in $t \in[0, T]$.

Proof: Throughout the proof $C$, will denote a generic constant. First we note that, due to the assumptions on the initial values and the coefficients in the equations, the error function is differentiable with respect to both $t$ and $x$ can be inserted in the system (4.1). Therefore $\{y, z\}$ is a genuine solution of the initial value problem (4.24)-(4.26).

The second term in right-hand side of Equation (4.25), $\partial_{t} \bar{w}_{1}$ can be written as follows: 


$$
\begin{gathered}
\partial_{t} \bar{w}_{1}=\mathscr{D}\left(\frac{\widehat{\gamma}}{2 \widehat{\lambda}} \partial_{x}\left(\sigma \bar{v}^{(2)}\right)\right) \\
=\underset{\mathfrak{D}}{2 \widehat{\lambda}} \partial_{x}\left[\sigma \left(G_{\mathscr{D}}(t) \stackrel{\circ}{v}-\epsilon \widehat{\gamma} G_{\mathscr{D}}(t) \partial_{x}\left(\frac{\sigma}{2 \widehat{\lambda}} \stackrel{\circ}{w}\right)\right.\right. \\
\left.\left.-\epsilon^{2} \frac{\widehat{\gamma}^{2}}{4} G_{\mathscr{D}}(t) \partial_{x}\left(\frac{\sigma}{\widehat{\lambda}^{2}} \partial_{x}(\sigma \stackrel{\circ}{v})\right)\right)\right]=\underset{\mathfrak{d}}{2 \widehat{\gamma}} \partial_{x}\left(G_{\mathscr{D}}(t) \stackrel{\circ}{v}\right)+\epsilon \bar{F}(t),
\end{gathered}
$$

where, utilizing identification (4.30) and assumptions (ii)-(iii), we have

$$
\|\bar{F}(t)\|_{L^{2}(\mathbb{R})} \leq C\left(1+\frac{1}{\sqrt{t}}\right) .
$$

To obtain the desired estimates we split the right-hand sides of (4.24) and (4.25) into two parts and accordingly we will be solving two problems:

Problem $P_{i}$ :

$$
\begin{gathered}
\partial_{t} y_{i}+\frac{\widehat{\gamma}}{\epsilon} \partial_{x}\left(\sigma z_{i}\right)=-\epsilon \partial_{x}\left(\sigma \widetilde{w}_{2}\right), \\
\partial_{t} z_{i}+\frac{\widehat{\gamma}}{\epsilon} \partial_{x}\left(\sigma y_{i}\right)+\frac{2 \widehat{\lambda}}{\epsilon} z_{i}=-\epsilon \widehat{\gamma} \partial_{x}\left(\sigma \widetilde{v}_{2}\right)-\epsilon^{2} \bar{F}
\end{gathered}
$$

with the initial value given by Equations (4.26).

Problem $P_{b}$ :

$$
\begin{gathered}
\partial_{t} y_{b}+\frac{\widehat{\gamma}}{\epsilon} \partial_{x}\left(\sigma z_{b}\right)=0, \\
\partial_{t} z_{b}+\frac{\widehat{\gamma}}{\epsilon} \partial_{x}\left(\sigma y_{b}\right)+\frac{2 \widehat{\lambda}}{\epsilon} z_{b}=-\epsilon \bar{F}_{1} \\
y_{b}(0)=0, \quad z_{b}(0)=0,
\end{gathered}
$$

where

$$
\bar{F}_{1}=\mathscr{D}\left[\frac{\widehat{\gamma}}{2 \widehat{\lambda}} \partial_{x}\left(G_{\mathscr{D}}(t) \stackrel{\circ}{v}\right)\right] .
$$

By the results of Section 2 we can write the solution $\left\{y_{i}, z_{i}\right\}$ as

$$
\begin{gathered}
{\left[\begin{array}{c}
y_{i}(t) \\
z_{i}(t)
\end{array}\right]=G_{\text {丁 }}(t)\left[\begin{array}{c}
y_{i}(0) \\
0
\end{array}\right]} \\
+\int_{0}^{t} G_{\text {丁 }}(t-s)\left[\begin{array}{c}
-\epsilon \partial_{x}\left(\sigma \widetilde{w}_{2}\left(s / \epsilon^{2}\right)\right) \\
-\epsilon \widehat{\gamma} \partial_{x}\left(\sigma \widetilde{v}_{2}\left(s / \epsilon^{2}\right)\right)-\epsilon^{2} \bar{F}(s)
\end{array}\right] d s
\end{gathered}
$$

and, since $\left(G_{\mathcal{G}}(t)\right)_{t \geq 0}$ is a semigroup of contractions in $\mathcal{H}_{\sigma}$, we have 


$$
\begin{aligned}
&\left\|\left[\begin{array}{c}
y_{i}(t) \\
z_{i}(t)
\end{array}\right]\right\|_{\mathcal{J G}_{\sigma}} \leq\left\|\left[\begin{array}{c}
y_{i}(0) \\
0
\end{array}\right]\right\|_{\mathcal{J G}_{\sigma}} \\
&+ \int_{0}^{t}\left\|\left[\begin{array}{c}
-\epsilon \partial_{x}\left(\sigma \widetilde{w}_{2}\left(s / \epsilon^{2}\right)\right. \\
-\epsilon \hat{\gamma} \partial_{x}\left(\sigma \widetilde{v}_{2}\left(s / \epsilon^{2}\right)-\epsilon^{2} \bar{F}(s)\right.
\end{array}\right]\right\|_{\mathcal{H G}_{\sigma}} d s \\
& \leq \epsilon^{2} C {\left[1+\int_{0}^{t / \epsilon^{2}} e^{-\hat{\lambda}_{0} s^{\prime}} Q\left(s^{\prime}\right) d s^{\prime}+M \int_{0}^{t}\left(1+\frac{1}{\sqrt{s}}\right) d s\right] } \\
& \leq C \epsilon^{2}(1+T+\sqrt{T}) .
\end{aligned}
$$

The constant $C$ depends on the coefficients and the initial values, $T$ is the length of the time interval, and $Q$ is a polynomial.

To estimate the solution of Problem $P_{b}$ we introduce an auxiliary function defined as a solution to the following problem:

$$
\begin{gathered}
\partial_{t} h=-\frac{2 \hat{\lambda}^{2}}{\epsilon} h+\epsilon \bar{F}_{1} \\
h(0)=0 .
\end{gathered}
$$

Clearly,

$$
h(t)=\epsilon \int_{0}^{t} e^{-\frac{2 \hat{\lambda}(t-s)}{\epsilon^{2}}} \bar{F}_{1}(s) d s
$$

and the pair $\left\{y_{b}, \widehat{z}_{b}\right\}$, where $\widehat{z}_{b}-h_{b}$, satisfies

$$
\begin{gathered}
\partial_{t} y_{b}+\frac{1}{\epsilon} \widehat{\gamma} \partial_{x}\left(\sigma \widehat{z}_{b}\right)=\widehat{\gamma} \partial_{x}\left(\sigma \int_{0}^{t} e^{-\frac{2 \widehat{\lambda}(t-s)}{\epsilon^{2}}} \bar{F}_{1}(s) d s\right) \\
\partial_{t} \widehat{z}_{b}+\frac{1}{\epsilon} \widehat{\gamma} \partial_{x}\left(\sigma y_{b}\right)+\frac{2 \widehat{\lambda}^{2}}{\epsilon^{2}} \widehat{z}_{b}=0 \\
y_{b}(0)=0, \widehat{z}_{b}(0)=0 .
\end{gathered}
$$

Using again the fact that the semigroup $\left(G_{\mathcal{G}}(t)\right)_{t \geq 0}$ is a contraction semigroup in ${ }^{3} 6_{\sigma}$ we obtain

$$
\left\|\left\{y_{b}, \widehat{z}_{b}\right\}\right\|_{\mathfrak{J G}_{\sigma}} \leq \int_{0}^{t}\left[\widehat{\gamma}\left\|\partial_{x}\left(\sigma \int_{0}^{t} e^{-\frac{2 \hat{\lambda}\left(s-s^{\prime}\right)}{\epsilon^{2}}} \bar{F}_{1}\left(s^{\prime}\right) d s^{\prime}\right)\right\|_{\mathcal{J G}_{\sigma}}\right] d s
$$




$$
\begin{gathered}
\leq C \int_{0}^{t} \int_{0}^{s^{\prime}}\left(1+\frac{s-s^{\prime}}{\epsilon^{2}}\right) e^{-\frac{2 \hat{\lambda}_{0}\left(s-s^{\prime}\right)}{\epsilon^{2}}} d s^{\prime} d s \\
\leq C\left(T \epsilon^{2}+\epsilon^{4}\right)
\end{gathered}
$$

since $\left\|G_{\mathfrak{D}}(t) \stackrel{\circ}{v}\right\|_{W_{2}^{k}(\mathbb{R})}$ is bounded for $k \leq 4$ (this statement can be proved similarly as the estimate (4.33).

We have also

$$
\|h(t)\|_{H_{\sigma}} \leq C \epsilon^{3}
$$

and, therefore, the mild solution $\left\{y_{i}+y_{b}, z_{i}+z_{b}\right\}$ to problem (4.24)-(4.26) satisfies the estimate

$$
\left\|\left\{y_{i}+y_{b}, z_{i}+z_{b}\right\}\right\|_{\mathfrak{H} 6} \leq C \epsilon^{2} .
$$

Since (4.24)-(4.26) has a classical solution $\{y, z\}$, this solution coincides with the mild solution and hence the estimate (4.31) is proved.

Remark 4.1: In the next section we shall need a sharper version of the estimate (4.31). It follows from Equation (4.32) that if we assume $\stackrel{\circ}{w} \in W_{2}^{4}(\mathbb{R})$, then the estimate (4.33) can be improved to

$$
\|\bar{F}(t)\|_{L^{2}(\mathbb{R})} \leq C\left(1+\frac{\epsilon}{\sqrt{t}}\right) .
$$

Hence, Equation (4.39) changes into

$$
\left\|\left[\begin{array}{l}
y_{i}(t) \\
z_{i}(t)
\end{array}\right]\right\|_{\mathcal{H}_{\mathcal{G}}} \leq \epsilon^{2}(1+T+\epsilon \sqrt{T})
$$

and, consequently, we have

$$
\left\|\left\{y_{i}+y_{b}, z_{i}+z_{b}\right\}\right\|_{\mathfrak{K} 6} \leq \epsilon^{2} C(\epsilon \sqrt{T}+T+\epsilon)
$$

where $T$ is the length of the time interval.

Remark 4.2: It is to be noted that the parameter $\epsilon^{2}$ was introduced in an artificial way to label the magnitude of the strength of correlations $\lambda\left(\lambda=\widehat{\lambda} / \epsilon^{2}\right)$ and, therefore, the error of the approximation is of order $\lambda$.

Formulas (4.22) and (4.23) together with Theorem 4.1 can be rewritten in the following form:

$$
\begin{gathered}
v=\bar{v}^{(2)}+\epsilon \widetilde{v}_{1}+\epsilon^{2} \widetilde{v}_{2}+O\left(\epsilon^{2}\right), \\
w=\widetilde{w}_{0}+\epsilon\left(\widetilde{w}_{1}+\bar{w}_{1}\right)+\epsilon^{2} \widetilde{w}_{2}+O\left(\epsilon^{2}\right) .
\end{gathered}
$$

We see that in fact, terms $\widetilde{v}_{2}$ and $\widetilde{w}_{2}$ are superfluous as they are multiplied by $\epsilon^{2}$. Similarly, if we are interested only in the error of approximation we can simplify the initial condition for the diffusion equation given by Equation (4.21) by dropping the last term. However, it follows (see [1], Chapter 5.7) that to get the required esti- 
mates we had to use the second order relation $\bar{w}_{2}=0$. The second order terms in approximation (4.47), (4.48) will be utilized later when we analyze the relation between the moments of correlated and uncorrelated random walks.

The observations made above can be summarized in the following corollary.

Corollary 4.1: Let $v$ be the density of the correlated random walk, which satisfies equations (4.1) and (4.2), let $\bar{v}^{(1)}$ be the solution of the problem

$$
\begin{gathered}
\partial_{t} \bar{v}^{(1)}=\frac{\gamma^{2}}{2} \partial_{x}\left(\frac{\sigma}{\lambda} \partial_{x}\left(\sigma \bar{v}^{(1)}\right)\right), \\
\bar{v}^{(1)}(0)=\stackrel{\circ}{v}-\epsilon \widehat{\gamma} \partial_{x}\left(\frac{\sigma}{2 \widehat{\lambda}} \stackrel{\circ}{w}\right),
\end{gathered}
$$

and let

$$
\tilde{v}_{1}(0)=\widehat{\gamma} \partial_{x}\left(\frac{\sigma}{2 \widehat{\lambda}} \stackrel{\circ}{w}\right)
$$

Then, for each $0<T<\infty$ there is a constant $C$ such that

$$
\left\|v(t)-\bar{v}^{(1)}(t)-\epsilon \widetilde{v}_{1}\left(t / \epsilon^{2}\right)\right\|_{L_{2}(\mathbb{R})} \leq C \epsilon^{2}
$$

uniformly for $t \in[0, T]$.

\section{Singularly Perturbed Systems: Second Case}

An intuitive perception of a correlated random walk is that one should be able to approximate it with a uncorrelated random walk whenever the correlations are weak. Hence, the additional assumption that

$$
\frac{\gamma^{2}}{\lambda}=O(1)
$$

which requires the velocity to go to infinity at a specified rate, seems to have no physical meaning and the question appears whether it is possible to obtain a diffusion approximation to the correlated random walk equation basic solely on the assumption $\lambda \rightarrow \infty$. Here we shall see that such an approximation is indeed possible and is of far better accuracy than that discussed in the previous section.

To obtain the singularly perturbed system corresponding to the assumption about the weakness of correlations we introduce $\widehat{\lambda}=\epsilon \lambda$ (so that now the strength of correlations is of order $\epsilon$ ) and consider the following system:

$$
\begin{gathered}
\partial_{t} v+\gamma \partial_{x}(\sigma w)=0 \\
\partial_{t} w+\partial_{x}(\sigma v)+\frac{2 \widehat{\lambda}}{\epsilon} w=0
\end{gathered}
$$

with the initial values given, as before, by Equations (4.2):

$$
v(0, x)=\stackrel{\circ}{v}, \quad w(0, x)=\stackrel{\circ}{w} .
$$

We can analyze this problem either directly, or notice that the substitution 


$$
t^{\prime}=\epsilon t
$$

reduces to (5.1) to (4.1), which was thoroughly examined in the previous section. Returning to the original variable $t$ we obtain from Equations (4.5)-(4.8) and (4.21) that

$$
\begin{gathered}
\partial_{t} \bar{v}^{(2)}=\frac{\epsilon \gamma^{2}}{2} \partial_{x}\left(\frac{\sigma}{\widehat{\lambda}} \partial_{x}\left(\sigma \bar{v}^{(2)}\right)\right) \\
\bar{v}^{(2)}(0)=\stackrel{\circ}{v}-\epsilon \gamma \partial_{x}\left(\frac{\sigma}{2 \widehat{\lambda}} \stackrel{o}{w}\right)-\epsilon^{2} \frac{\gamma^{2}}{4} \partial_{x}\left(\frac{\sigma}{\widehat{\lambda}^{2}} \partial_{x}(\sigma \stackrel{\circ}{v})\right)
\end{gathered}
$$

and

$$
\begin{gathered}
\bar{w}_{0}=\bar{w}_{2} \equiv 0 \\
\bar{w}_{1}=-\frac{\gamma}{2 \widehat{\lambda}} \partial_{x}\left(\sigma \bar{v}^{(2)}\right) .
\end{gathered}
$$

The only difference is that the parameter $\epsilon$ enters the diffusion coefficient or, in other words, that the diffusion coefficient is of order $1 / \lambda=\epsilon / \widehat{\lambda}$, which seems to be a natural result.

The initial layer terms given by Equations (4.12) do not change the form; the only difference is that $\tau=\theta / \epsilon^{2}=t / \epsilon$.

The equations for the error has a form similar to Equations (4.22), (4.23):

$$
\begin{gathered}
y(t)=v(t)-\bar{v}^{(2)}(t)-\epsilon \widetilde{v}_{1}(t / \epsilon)-\epsilon^{2} \widetilde{v}_{2}(t / \epsilon) \\
z(t)=w(t)-\widetilde{w}_{0}(t / \epsilon)-\epsilon\left(\widetilde{w}_{1}(t)+\bar{w}_{1}(t / \epsilon)\right)-\epsilon^{2} \widetilde{w}_{2}(t / \epsilon),
\end{gathered}
$$

where this time $\bar{v}^{(2)}$ is a solution to problem (5.2).

To obtain an estimate of the error (5.4), (5.5) we note that estimate (4.31) (and (4.46)), obtained after substitution $t^{\prime}=\epsilon t$, is valid for each $t^{\prime}$ so that we can safely replace $t^{\prime}$ by $t$ and get te required estimates in $t$. Moreover, if $0 \leq t \leq T$, then $0 \leq t^{\prime} \leq$ $\epsilon T$, and from Equation (4.46) we see that the error is of order $\epsilon^{3}$. Hence, the following theorem is true.

Theorem 5.1: Let

(i) $\quad \sigma$ and $\hat{\lambda}$ satisfy

$$
\begin{aligned}
& 0<\sigma_{0} \leq \sigma(x) \leq \sigma_{\infty}<\infty \\
& 0<\widehat{\lambda}_{0} \leq \widehat{\lambda}(x) \leq \widehat{\lambda}_{\infty}<\infty
\end{aligned}
$$

(ii) $\quad \stackrel{\circ}{v} \in W_{2}^{4}(\mathbb{R})$,

(iii) $\stackrel{\circ}{w} \in W_{2}^{4}(\mathbb{R})$.

Then, for each $T, 0 \leq T \leq \infty$, there is a constant $C$ such that the error of approximation $\{y, z\}$ defined by Equations (5.4) and (5.5) satisfies

$$
\|\{y, z\}\|_{L_{2}(\mathbb{R}) \times L_{2}(\mathbb{R})} \leq C \epsilon^{2}
$$


uniformly in $t \in[0, T]$.

Note that in this case, the error of diffusion approximation is of order of $1 / \lambda^{3}$, which is considerably better than for singularly perturbed system (4.1).

Remark 5.1: If we select the direct way of obtaining estimate (5.6) and proceed as in the previous section, we obtain the following equations for the error

$$
\begin{gathered}
\partial_{t} y+\gamma \partial_{x}(\sigma z)=-\epsilon^{2} \partial_{x}\left(\sigma \widetilde{w}_{2}\right) \\
\partial_{t} z+\gamma \partial_{x}(\sigma y)+\frac{2 \widehat{\lambda}}{\epsilon} z=-\epsilon^{2} \gamma \partial_{x}\left(\sigma \widetilde{v}_{2}\right)-\epsilon \partial_{t} \bar{w}_{1} .
\end{gathered}
$$

However, by Equations (5.3) and (5.2) we obtain

$$
\begin{gathered}
\partial_{t} \bar{w}_{1}=-\frac{\gamma}{2 \widehat{\lambda}} \partial_{x}\left(\sigma \partial_{t} \bar{v}^{(2)}\right) \\
=\epsilon \frac{\gamma^{2}}{2 \widehat{\lambda}} \partial_{x}\left[\sigma \partial_{x}\left(\sigma \bar{w}_{1}\right)\right]
\end{gathered}
$$

and the bulk part term in Equation (5.8) is multiplied by $\epsilon^{2}$. Now, following the same procedure as when estimating the solution of Problem $P_{b}$ in Theorem 4.1 , we show that the contribution of term $\epsilon \bar{w}_{1}$ to the total error is of order $\epsilon^{3}$.

\section{Application to Random Walk Theory}

Let us return to the real coefficients $\gamma$ and $\lambda$ and remove $\epsilon$ from the formulas approximating the density $v$ of the correlated random walk. It turns out that in both cases we obtain the same equations

$$
v \approx v^{(2)}=\bar{v}^{(2)}+\widetilde{v}_{1}^{\prime}+\widetilde{v}_{2}^{\prime}
$$

where $\bar{v}^{(2)}$ satisfies

$$
\begin{gathered}
\partial_{t} \bar{v}^{(2)}=\frac{\gamma^{2}}{2} \partial_{x}\left(\frac{\sigma}{\lambda} \partial_{x}\left(\sigma \bar{v}^{(2)}\right)\right) \\
\bar{v}^{(2)}(0)=\stackrel{\circ}{v}-\gamma \partial_{x}\left(\frac{\sigma}{2 \lambda} \stackrel{\circ}{w}\right)-\frac{\gamma^{2}}{4} \partial_{x}\left(\frac{\sigma}{\lambda^{2}} \partial_{x}(\sigma \stackrel{\circ}{v})\right),
\end{gathered}
$$

and

$$
\begin{gathered}
v_{1}^{\prime}(t)=\epsilon \tilde{v}_{1}(\tau)=\gamma \partial_{x}\left(\frac{\sigma}{2 \lambda} e^{-2 \lambda t} \stackrel{\circ}{w}\right) \\
\tilde{v}_{2}^{\prime}(t)=\epsilon^{2} \widetilde{v}_{2}(\tau)=\gamma^{2} \partial_{x}\left(\frac{\sigma}{4 \lambda^{2}} e^{-2 \lambda t} \partial_{x}(\sigma \stackrel{\circ}{v})\right) .
\end{gathered}
$$

We recall that the profound difference between the two asymptotic expansion is that, when $\lambda \rightarrow \infty$ but $\lambda / \gamma^{2}$ remains finite, the error of approximation (6.1) is of order $1 / \lambda$ and if we require only $\lambda \rightarrow \infty$, then the error is of order $1 / \lambda^{3}$.

To show clearly the meaning of the asymptotic expansions obtained in previous sections in random walk theory we assume throughout this section that the coefficients of the original telegraph system (2.15) are independent of $x$ and demonstrate 
that in such a case, the moments of $v$ up to the second order (and, therefore, the variance of $v$ ) are exactly equal to the respective moments of $v^{(2)}$.

For a given function $f$ and $p=0,1, \ldots$, we denote

$$
\langle f\rangle_{p}=\int_{\mathbb{R}} x^{p} f(x) d x .
$$

We have the following theorem.

Theorem 6.1: Let $\stackrel{\circ}{v}$ and $\stackrel{\circ}{w}$ satisfy the assumptions of Theorem 4.1 and, moreover,

$$
x^{k} \partial_{x}^{j} \stackrel{\circ}{v}, x^{k} \partial_{x}^{j} \stackrel{\circ}{w} \in L_{2}(\mathbb{R})
$$

for $k=1,2,3$ and $j=0,1$. Then, for all $t \geq 0$ and $p=0,1,2$,

$$
\langle v\rangle_{p}(t)=\left\langle v^{(2)}\right\rangle_{p}(t)
$$

where $v^{(2)}$ is defined by Equation (6.1).

Proof: Using the Fourier transform one can verify that if the initial values have the properties specified in the theorem, then the same properties will have the solutions $(v, w)$ to the telegraph system

$$
\begin{gathered}
\partial_{t} v+\gamma \sigma \partial_{x} w=0, \\
\partial_{t} w+\gamma \sigma \partial_{x} v+2 \lambda w=0, \\
v(0)=\stackrel{\circ}{v}, \quad w(0)=\stackrel{\circ}{w},
\end{gathered}
$$

and the solution $\bar{v}^{(2)}$ to diffusion equation (6.2). Then, the moments of the solutions will exist up to the second order since, e.g.,

$$
\begin{gathered}
\int_{\mathbb{R}} x^{2} v(t, x) d x \leq \int_{\mathbb{R}}(1+|x|)^{-1}(1+|x|) v(t, x) d x \\
\leq\left(\int_{\mathbb{R}}(1+|x|)^{-2} d x\right)^{1 / 2}\left(\int_{\mathbb{R}}(1+|x|)^{2}|v(t, x)|^{2} d x\right)^{1 / 2}<\infty .
\end{gathered}
$$

Since $x^{k} \partial_{x}^{j} v$ are integrable for $k=0,1,2$ and $j=0,1$ and continuous on $\mathbb{R}$, by the Sobolev imbedding theorem, we have

$$
\lim _{x \rightarrow \pm \infty} x^{k} \partial_{x}^{j} v=0
$$

Similar results hold for $w$ and $\bar{v}^{(2)}$, where in the last case we can have $0 \leq j \leq 3$. The above assumptions ensure also that all the moments are differentiable with respect to $t$ as $L_{1}$-valued function; thus, we can interchange operations $\partial_{t}$ and $\int_{\mathbb{R}}$.

Using these facts we obtain the following equations for the moments of the systems (6.7): 


$$
\begin{gathered}
\partial_{t}\langle v\rangle_{0}=0,\langle v\rangle_{0}(0)=\langle\stackrel{\circ}{v}\rangle_{0}, \\
\partial_{t}\langle w\rangle_{0}=-2 \lambda\langle w\rangle_{0}\langle w\rangle_{0}(0)=\langle\stackrel{\circ}{w}\rangle_{0}, \\
\partial_{t}\langle v\rangle_{1}=\gamma \sigma\langle w\rangle_{0}, \quad\langle v\rangle_{1}(0)=\langle\stackrel{\circ}{v}\rangle_{1}, \\
\partial_{t}\langle w\rangle_{1}=-2 \lambda\langle w\rangle_{1}+\gamma \sigma\langle v\rangle_{0},\langle w\rangle_{1}(0)=\langle\stackrel{\circ}{w}\rangle_{1}, \\
\partial_{t}\langle v\rangle_{2}=2 \gamma \sigma\langle w\rangle_{1},\langle v\rangle_{2}(0)=\langle\stackrel{\circ}{v}\rangle_{2} .
\end{gathered}
$$

Solving these equations we obtain

$$
\begin{gathered}
\langle v\rangle_{0}(t)=\langle\stackrel{\circ}{v}\rangle_{0}, \\
\langle w\rangle_{0}(t)=\langle\stackrel{\circ}{w}\rangle_{0} e^{-2 t} \\
\langle v\rangle_{1}(t)=-\frac{\gamma \sigma\langle\stackrel{\circ}{w}\rangle_{0}}{2 \lambda} e^{-2 \lambda t}+\frac{\gamma \sigma\langle\stackrel{\circ}{w}\rangle_{0}}{2 \lambda}+\langle\stackrel{\circ}{v}\rangle_{1}, \\
\langle w\rangle_{1}(t)=\left(-\frac{\gamma \sigma\langle\stackrel{\circ}{v}\rangle_{0}}{2 \lambda}+\langle\stackrel{\circ}{w}\rangle_{1}\right) e^{-2 \lambda t}+\frac{\gamma \sigma\langle\stackrel{\circ}{v}\rangle_{0}}{2 \lambda} \\
\langle v\rangle_{2}(t)=\frac{\gamma \sigma\langle\stackrel{\circ}{w}\rangle_{0}}{2 \lambda} t+\left(-\frac{\gamma \sigma\langle\stackrel{\circ}{w}\rangle_{1}}{\lambda}+\frac{\gamma^{2} \sigma^{2}\langle\stackrel{\circ}{v}\rangle_{0}}{2 \lambda^{2}}\right) e^{-2 \lambda t} \\
+\frac{\gamma \sigma\langle\stackrel{\circ}{w}\rangle_{1}}{\lambda}-\frac{\gamma^{2} \sigma^{2}\langle\stackrel{\circ}{v}\rangle_{0}}{2 \lambda^{2}}+\langle\stackrel{\circ}{v}\rangle_{2} .
\end{gathered}
$$

The moments of $\bar{v}^{(2)}$ can be calculated as follows:

$$
\begin{gathered}
\partial_{t}\left\langle\bar{v}^{(2)}\right\rangle_{0}=0,\left\langle\bar{v}^{(2)}\right\rangle_{0}(0)=\langle\stackrel{o}{v}\rangle_{0} \\
\partial_{t}\left\langle\bar{v}^{(2)}\right\rangle_{1}=0,\left\langle\bar{v}^{(2)}\right\rangle_{1}(0)=\langle\stackrel{\circ}{v}\rangle_{1}+\frac{\gamma \sigma}{2 \lambda}\langle\stackrel{\circ}{w}\rangle_{0} \\
\partial_{t}\left\langle\bar{v}^{(2)}\right\rangle_{2}=\frac{\gamma^{2} \sigma^{2}}{\lambda}\left\langle\bar{v}^{(2)}\right\rangle_{0} \\
\left\langle\bar{v}^{(2)}\right\rangle_{2}(0)=\langle\stackrel{\circ}{v}\rangle_{2}+\frac{\gamma \sigma\langle\stackrel{o}{w}\rangle_{1}}{\lambda}-\frac{\gamma^{2} \sigma^{2}\langle\stackrel{o}{v}\rangle_{0}}{2 \lambda^{2}}
\end{gathered}
$$

which gives

$$
\begin{gathered}
\left\langle\bar{v}^{(2)}\right\rangle_{0}(t)=\langle\stackrel{\circ}{v}\rangle_{t} \\
\left\langle\bar{v}^{(2)}\right\rangle_{1}(t)=\langle\stackrel{\circ}{v}\rangle_{1}+\frac{\gamma \sigma}{2 \lambda}\langle\stackrel{\circ}{w}\rangle_{0} \\
\left\langle\bar{v}^{(2)}\right\rangle_{2}(t)=\frac{\gamma^{2} \sigma^{2}\langle\stackrel{\circ}{v}\rangle_{0}}{\lambda} t+\langle\stackrel{\circ}{v}\rangle_{2}+\frac{\gamma \sigma\langle\stackrel{\circ}{w}\rangle_{1}}{\lambda}-\frac{\gamma^{2} \sigma^{2}\langle\stackrel{o}{v}\rangle_{0}}{2 \lambda^{2}} .
\end{gathered}
$$

The moments of the initial layer terms are given by 


$$
\begin{gathered}
\left\langle v_{1}^{\prime}\right\rangle_{0}(t)=\left\langle\widetilde{v}_{2}^{\prime}\right\rangle_{0}(t)=0, \\
\left\langle\widetilde{v}_{1}^{\prime}\right\rangle_{1}(t)=-\frac{\gamma \sigma\langle\stackrel{o}{w}\rangle_{0}}{2 \lambda} e^{-2 \lambda t} \\
\left\langle\widetilde{v}_{2}^{\prime}\right\rangle_{1}(t)=0, \\
\left\langle\widetilde{v}_{1}^{\prime}\right\rangle_{2}(t)=-\frac{\gamma \sigma\langle\stackrel{o}{w}\rangle_{1}}{\lambda} e^{-2 \lambda t} \\
\left\langle\widetilde{v}_{2}^{\prime}\right\rangle_{2}(t)=\frac{\gamma^{2} \sigma^{2}\langle\stackrel{o}{v}\rangle_{0}}{2 \lambda^{2}} e^{-2 \lambda t},
\end{gathered}
$$

Adding Equations (6.11), (6.14) and (6.15) we see that for all $t \geq 0$ we obtain

$$
\left\langle v^{(2)}\right\rangle_{1}(t)=\langle v\rangle_{1}(t)
$$

where $\langle v\rangle_{1}$ is given by Equation (6.9), and adding equations (6.12), (6.16) and (6.17), we obtain that

$$
\left\langle v^{(2)}\right\rangle_{2}(t)=\langle v\rangle_{2}(t)
$$

where $\langle v\rangle_{2}$ is given by Equation (6.10). Hence, the theorem is proved.

\section{Acknowledgements}

The support of the Foundation for Research Development and the University of Natal Research Fund is acknowledged.

\section{References}

[1] Banasiak, J., Singular perturbations of resonance type with applications to the kinetic theory, In: Proc. of the Conf. on Evolution Equations, Glasgow 1994, Longman, Harlow, Essex (1995), 53-67.

[2] Banasiak, J., Asymptotic analysis of the linear Boltzmann equation with and without an external field: Comparison of various approaches, Transport Theory and Statistical Physics 26:182 (1997), 103-119.

[3] Banasiak, J. and Mika, J.R., Asymptotic analysis of the Fokker-Planck equation of Brownian motion, Math. Models and Methods in Appl. Sci. 4:1 (1994), 17-33.

[4] Banasiak, J. and Mika, J.R., Diffusion limit for the Boltzmann equation of the neutron transport theory, Math. Methods in the Applied Sciences 17 (1994), 1071-1087.

[5] Goldstein, J.E., Semigroups of Linear Operators and Applications, Oxford University Press, New York 1985.

[6] Goldstein, S., On diffusion by discontinuous movements and the telegraph equation, Quarterly J. of Mech. and Appl. Math. 4 (1951), 129-156.

[7] Janssen, A., The distance between the Kac process and the Wiener process with applications to generalized telegraph equations, $J$. of Theoretical Prob. 3:2 
(1990), 349-360.

[8] Kac, M., A stochastic model related to the telegrapher's equation, Rocky Mt. J. Math 4 (1974), 497-509.

[9] Mika, J.R., New asymptotic expansion algorithm for singularly perturbed evolution equations, Math. Methods in Appl. Sciences 3 (1981), 172-188.

[10] Mika, J.R. and Banasiak, J., Diffusion limit for a linear kinetic equation, Trans. Theory and Stat. Physics 24:1-3 (1995), 41-53.

[11] Mika, J.R. and Banasiak, J., Singularly Perturbed Evolution Equations with Applications to Kinetic Theory, World Scientific, Singapore 1995.

[12] Mika, J.R. and Palczewski, A., Asymptotic analysis of singularly perturbed systems of ordinary differential equations, Computers and Mathematics with Applications 21:10 (1991), 13-32.

[13] Mika, J.R.and Stankiewicz, R., Asymptotic equivalence of the $P_{1}$ and diffusion equations, Trans. Theory and Statistical Physics 7:3 (1978), 81-101.

[14] Mika, J.R. and Stankiewicz, R., Asymptotic relationship between telegraphic and diffusion equations, Math. Methods in the Applied Sciences 3 (1981), 21-37.

[15] Taylor, G.I., Diffusion by continuous movements, Proc. of London Mathematical Society 20 (1920), 196-212.

[16] Zauderer, E., Partial Differential Equations of Applied Mathematics, 2nd ed., John Wiley \& Sons, New York 1988. 


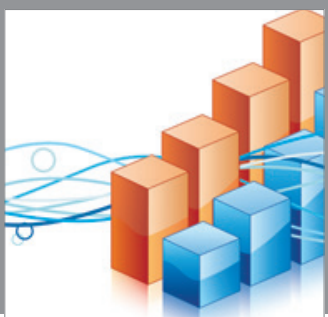

Advances in

Operations Research

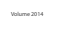

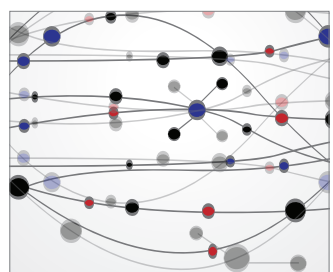

\section{The Scientific} World Journal
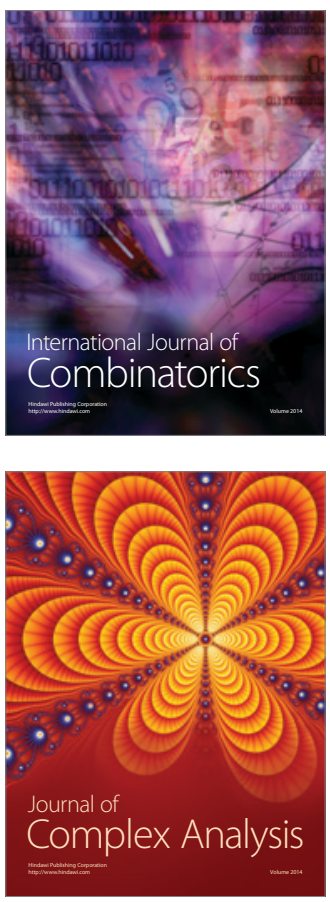

International Journal of

Mathematics and

Mathematical

Sciences
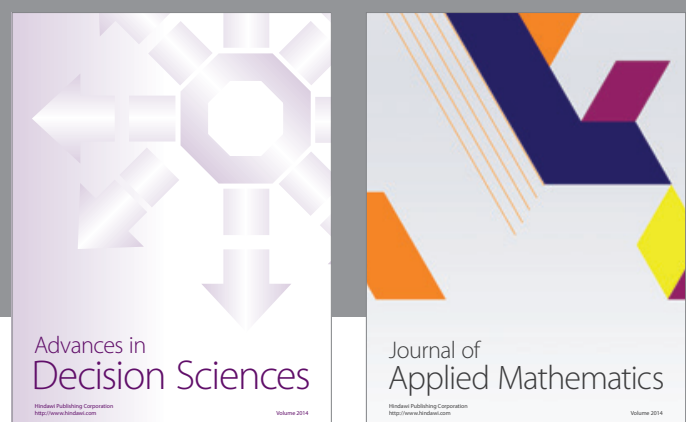

Journal of

Applied Mathematics
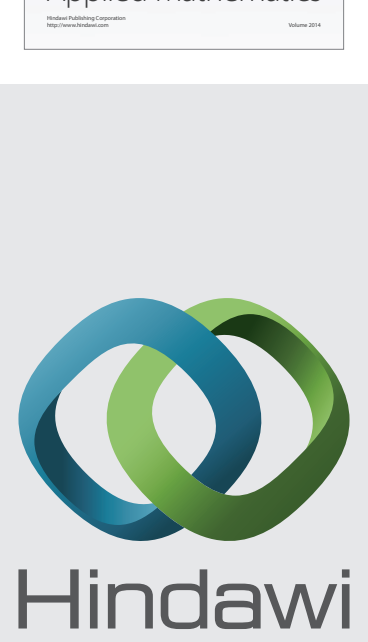

Submit your manuscripts at http://www.hindawi.com
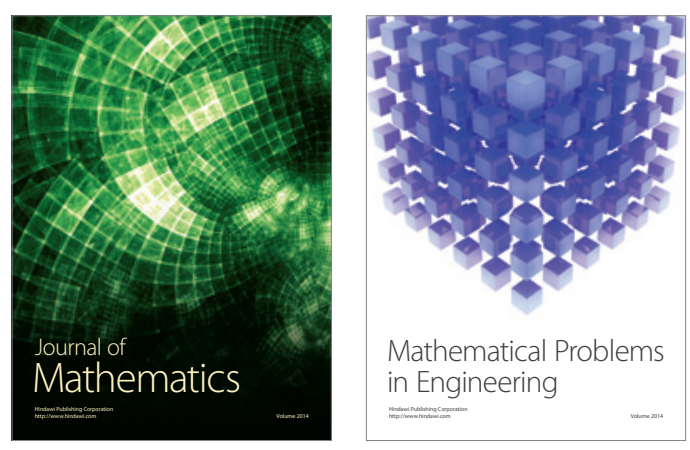

Mathematical Problems in Engineering
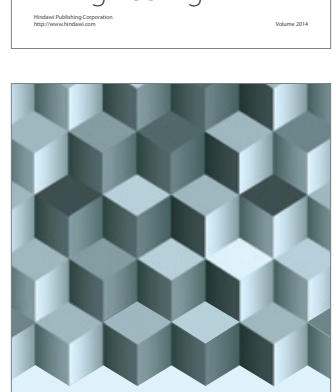

Journal of

Function Spaces
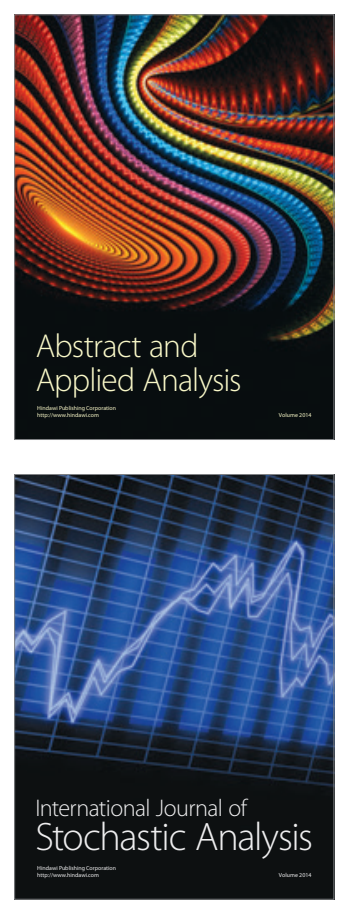

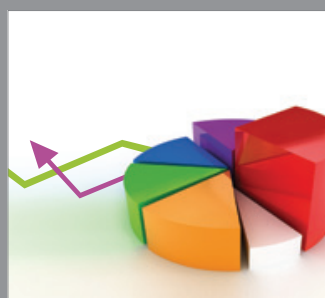

ournal of

Probability and Statistics

Promensencen
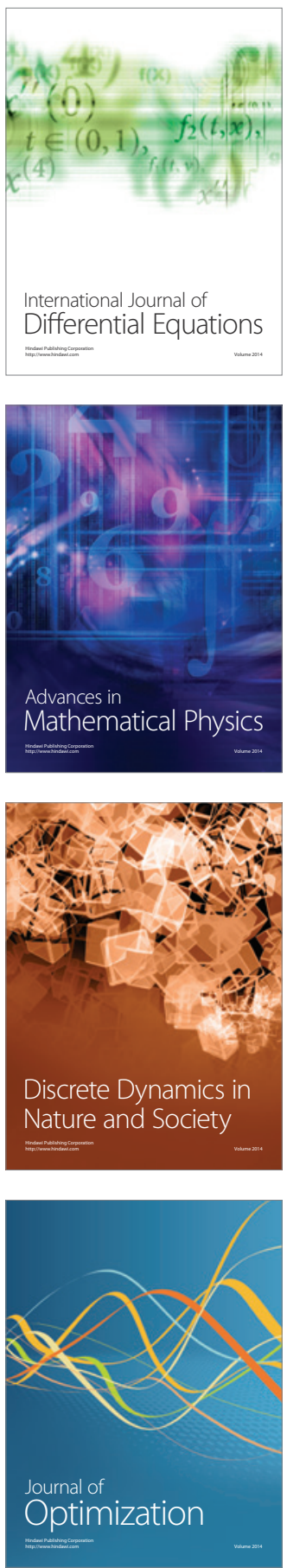